\title{
A Comprehensive Study on Crosstalk Suppression Techniques in Fiber Optical Parametric Amplifier by Modulation Format
}

\author{
Bill Ping-Piu Kuo, Student Member, IEEE, P. C. Chui, and Kenneth Kin-Yip Wong, Member, IEEE
}

\begin{abstract}
With the presence of multiple-WDM input signals, ON-OFF keying (OOK)-modulated signals suffer from crosstalk in fiber optical parametric amplifier (OPA) due to cross-gain modulation (XGM) and four-wave mixing (FWM) effects. We demonstrated substantial crosstalk suppression in one-pump OPA by using return-zero differential phase-shift keying (RZ-DPSK) modulation format, which with its pattern-independent amplitude and subunity duty cycle would be effective in reducing the XGM and FWM effects significantly. By using the RZ-DPSK format, the power penalty was improved by at least $0.8 \mathrm{~dB}$ over $\mathrm{RZ}-\mathrm{OOK}$, non-RZ (NRZ)-DPSK, and NRZ-OOK formats with four $10 \mathrm{~Gb} / \mathrm{s}$ channels, separated by $200 \mathrm{GHz}$ spacing. With eight $10 \mathrm{~Gb} / \mathrm{s}$ channels separated by $100 \mathrm{GHz}$ spacing, a $Q$-factor penalty of the RZ-DPSK signal was reduced by $2.4 \mathrm{~dB}$ compared to $\mathrm{RZ}-\mathrm{OOK}$ counterparts.
\end{abstract}

Index Terms-Cross-gain modulation (XGM), four-wave mixing (FWM), modulation format, optical crosstalk, optical parametric amplifier (OPA).

\section{INTRODUCTION}

I N MODERN optical network, dense wavelength-division multiplexing (DWDM) has proven to be an effective way to increase the capacity of existing infrastructure. Nowadays, reamplification of DWDM signals mainly relies on erbiumdoped fiber amplifiers (EDFAs) and Raman amplifiers (RAs), which can provide high signal gain with low noise figure. On top of these two types of amplifiers, fiber-based optical parametric amplifiers (OPAs), which can provide wide gain bandwidth [1], high signal gain, [2] and low noise figure [3], and polarizationinsensitive operation by polarization diversity [4], [5] and orthogonal pumping [6]-[8], has received extensive research interest for deployment in WDM links. However, as reported in previous research, ON-OFF keying (OOK)-modulated WDM signals amplified by one-pump OPA suffer from crosstalk distortion due to cross-gain modulation (XGM) [9], [10] and fourwave mixing (FWM) effects [9], [11]-[13]. Although crosstalk suppression techniques for two-pump OPAs have been demonstrated using orthogonal pumps [14] and channel polarization interleaving [15], [16], these techniques cannot be applied to

Manuscript received November 3, 2007; revised December 28, 2007. This work was supported in part by the Research Grants Council of the Hong Kong Special Administrative Region, China, under a grant to Project HKU 7179/06E).

The authors are with Photonics Systems Research Laboratory, Department of Electrical and Electronic Engineering, the University of Hong Kong, Hong Kong, SAR (e-mail: ppkuo@eee.hku.hk; pcchui@eee.hku.hk; kywong@eee.hku.hk).

Color versions of one or more of the figures in this paper are available online at http://ieeexplore.ieee.org.

Digital Object Identifier 10.1109/JSTQE.2008.920032 one-pump OPAs that have gain bandwidth that two-pump OPAs still cannot provide [1], [17]. Even though a crosstalk suppression technique based on reduced fiber length can be utilized in one-pump OPA in principle, [18], the available gain and bandwidth are sacrificed, or the utilization level of pump power has to be reduced. On the other hand, as parametric amplification is format-transparent, crosstalk suppression via wise choice of modulation format could be applied to both type of OPA and even incorporated with other crosstalk suppression techniques to further enhance the performance of the OPA in WDM systems.

Previously, crosstalk suppression via differential phase-shift keying (DPSK) modulation format has been demonstrated in semiconductor optical amplifiers (SOAs) and fiber-based wavelength converter to mitigate cross-gain modulation (XGM) penalty [19], [20]. Previous studies have also verified the advantage of using DPSK format in OPA for XGM-crosstalk suppression with multiwavelength input [21], [22], and in deeply saturated OPA with single-channel configuration [23]. Moreover, a previous theoretical study has shown that modulation formats with return-zero (RZ) pulse shape have higher immunity to FWM-induced crosstalk over nonreturn-zero (NRZ) counterpart because of pulse walkoff between channels [24]. Combining the advantages of DPSK format and RZ pulse shape, RZ-DPSK modulation formats should be a promising candidate for alleviating crosstalk in OPA.

In this paper, we present, for the first time to the best of our knowledge, a comprehensive study on the robustness of commonly used modulation formats, including OOK and DPSK formats with RZ and NRZ pulse shapes, to crosstalk in the OPA. The remaining sections of this paper are organized as follows. Section II presents the origin of signal crosstalk in the OPA and the justification of suppressing crosstalk by means of modulation format. Section III shows the one-pump OPA test bench used to investigate the immunity of signals with different modulation formats to crosstalk in the OPA. The results with wide and narrow channel spacing are discussed in Section IV. Concluding remarks will be given in Section V.

\section{THEORY}

The origin of crosstalk between WDM channels due to XGM and FWM in the OPA is illustrated in Fig. 1. Only channel \#1 $\left(\lambda_{1}\right)$ is shown with intensity modulation (IM) for clarity. Also note that the corresponding idlers for all four channels $\left(\lambda_{1}-\lambda_{4}\right)$ have not been shown here for simplicity. The signal $\left(\lambda_{1}\right)$ is amplified by the parametric gain (with pump wavelength at $\lambda_{P}$ ). As 


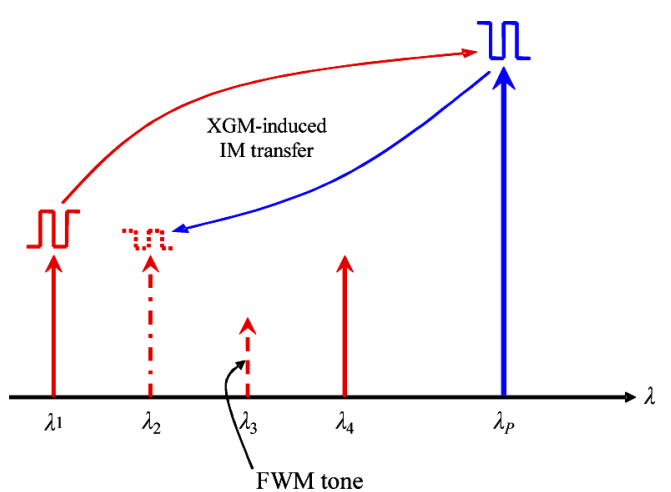

Fig. 1. Illustration of signal crosstalk due to XGM and FWM in 1P-OPA.

the signal is amplified, power is drawn from the pump because the total optical power remains constant. As a result, the pump itself now contains IM and all channels \#2-\#4 exhibit timevarying gain, while the instantaneous gain depends on whether they travel with a depleted part of the pump, or an undepleted one. As a result, the amplified channels \#2-\#4 themselves exhibit IM, which constitutes crosstalk. If we now consider that channels \#2-\#4 are replaced by independent modulation signals, it is clear that their amplitudes will experience fluctuations due to XGM crosstalk induced by the first channel and vice versa, which will lead to deterioration of their qualities. Furthermore, when the channels copropagate through the nonlinear gain medium of the OPA, they take part in signal-signal FWM process and generate spurious FWM tones [25]. If the channels are spaced equally in frequency domain, the FWM tones will lie on the same frequencies of other victim channels, for example, channel \#3 in Fig. 1. In this case, the FWM tones will generate beat noise on the victim channels and result in FWM crosstalk.

To reduce the extent of signal crosstalk in the OPA, a viable approach is to select a modulation format that can reduce the interactions of signal in the OPA. RZ-DPSK modulation format would be a promising candidate to alleviate signal interactions for two reasons. First, as for any DPSK format, the amplitude of the RZ-DPSK signal is data-pattern independent. As a result, there will be no intensity-modulation transfer to the adjacent channels through the XGM, and thus XGM crosstalk can be relieved. Second, thanks to shorter pulse duration of RZ formats, the RZ-DPSK signal pulse has shorter interaction length than other NRZ formats in the presence of dispersion, which in turn reduces the strength of signal-signal FWM processes. In the following sections, we will experimentally demonstrate the advantage of using the RZ-DPSK format in the OPA over other commonly used modulation formats.

\section{ONE-PUMP-OPA TEST BENCH}

The experimental setup for one-pump OPA used in this study is shown in Fig. 2. The nonlinear medium used for parametric amplification was a $1 \mathrm{~km}$ spool of highly-nonlinear dispersionshifted fiber (HNL-DSF) with zero-dispersion wavelength $\lambda_{0} \approx$ $1560 \mathrm{~nm}$, dispersion slope $d D / d \lambda \approx 0.024 \mathrm{ps} / \mathrm{nm}^{2} / \mathrm{km}$, and nonlinear coefficient $\gamma=14 / \mathrm{W} / \mathrm{km}$. The transmitter module

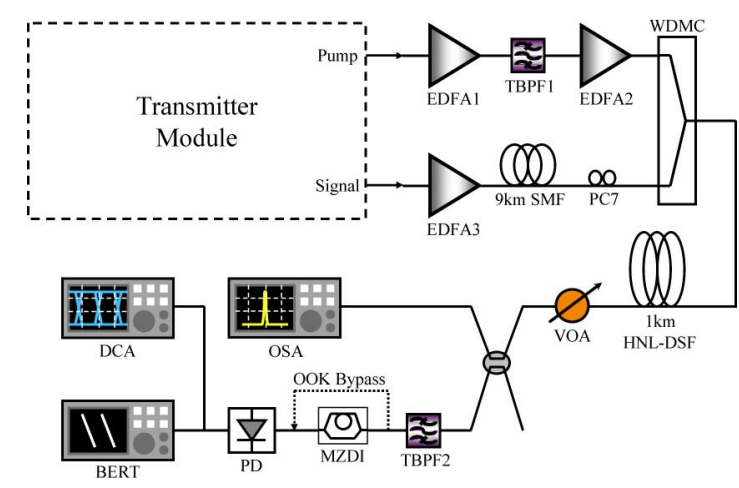

Fig. 2. 1P-OPA with DPSK/OOK signals. VOA: Variable optical attenuator; BERT: bit-error rate tester. Refer to the text for a detailed description of the experimental setup.

served as a signal source with selectable modulation format, and OPA pump source with phase-dithering for suppressing stimulated Brillouin scattering (SBS) in the HNL-DSF. After the transmitter module, the pump was boosted by two stages of EDFAs (EDFA1 and EDFA2), with a tunable band pass filter (TBPF1) inserted in between to suppress amplified spontaneous emission (ASE) level at the EDFA2 input. On the other hand, the signals were amplified by EDFA3, and subsequently, decorrelated by a spool of 9-km SMF-28 fiber. The amplified pump and signal were then combined and launched into the HNL-DSF for parametric amplification. The state of polarizations (SOPs) of signals were aligned to that of the pump through polarization controller PC7 in order to maximize signal gain. The output spectrum from HNL-DSF was then monitored through optical spectrum analyzer (OSA), whereas the signals were demodulated with a Mach-Zehnder delay interferometer (MZDI) and detected using a single-ended photodetector (PD) for DPSK signals, or directly detected by a photodetector (PD) for OOK signals.

\section{RESUlTS AND DisCUSSION}

\section{A. Preliminary Studies With Wide Channel Spacing}

In order to find out the best modulation format in the OPA, a preliminary comparison was performed with four formats, namely, RZ-DPSK, RZ-OOK, NRZ-DPSK, and NRZ-OOK. The transmitter module for this comparison is shown in Fig. 3. Four tunable signal laser sources (TLS1-4), with wavelengths set at 1567.7, 1569.3, 1570.9, and $1572.5 \mathrm{~nm}$, were combined and launched into the Mach-Zehnder modulator MZM1. The modulating signal to MZM1 was selected from a $10-\mathrm{GHz}$ clock signal for RZ pulse carving in case of RZ formats, or a DC bias for NRZ formats. The generated RZ pulses had a duty ratio of $48 \%$. The signals after MZM1 were routed into different modulators for DPSK or OOK modulation. In DPSK modulator, as shown in Fig. 3(a), the signals were combined with the pump from a DFB laser (DFB1) at $1561.3 \mathrm{~nm}$ and phase modulated by $2^{31}-1$ pseudorandom binary sequence (PRBS) at phase modulator (PM). Note that the same PM was deployed for signal data modulation and pump phase dithering simultaneously. Temporal alignment between the signal pulse train and 


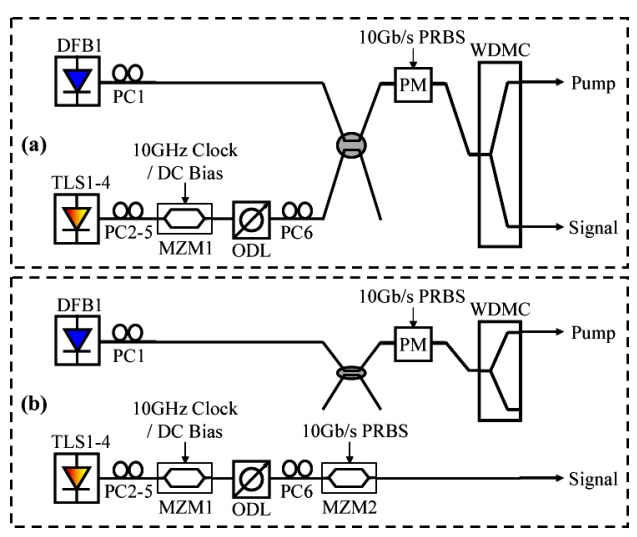

Fig. 3. Four channels, 200-GHz channel spacing transmitter modules for (a) DPSK and (b) OOK modulation format.

modulating signal fed into the PM was done by adjusting the tunable optical delay line (ODL) in signal path. The modulated signals and pumps were then decoupled using a WDM coupler (WDMC). In the OOK modulator, as shown in Fig. 3(b), the signals were intensity modulated at another intensity modulator MZM2 driven by $2^{31}-1$ PRBS for data modulation, while the pump was independently phase dithered at the PM by $2^{31}-1$ PRBS. The unused couplers on the pump path in OOK modulators were not removed to keep the input power of pump to the pump amplifiers the same as with DPSK modulator, and therefore, maintained the optical SNR (OSNR) of the pump. The pump was then amplified to $27 \mathrm{dBm}$ while the signal was boosted to total power of $1.83 \mathrm{dBm}$ at HNL-DSF input, as shown in Fig. 2. The gain attained at this input signal power was 18 $\mathrm{dB}$, while the small-signal gain was $24 \mathrm{~dB}$. The gain saturation suggested that significant pump depletion occurred at this signal power level.

Fig. 4 shows the eye diagrams of signal at channel \#1 modulated with different formats. Significant eye distortion is observable for OOK-modulated signals (Fig. 4(c) and (d) on the right column). Because of XGM crosstalk, multiple-mark-level features are seen from RZ-OOK eyes [Fig. 4(c)]. Such features are not clearly observable in NRZ-OOK eyes [Fig. 4(d)] as beat noise overwhelmed XGM-induced distortion in the NRZ-OOK signal. On the other hand, clear eye opening can be obtained with DPSK signals. Higher noise level, as observed from the eye of the NRZ-DPSK signal, was mainly due to higher FWM-induced crosstalk level.

In order to investigate the FWM-induced crosstalk level, the FWM-tone powers were measured with one signal channel switched OFF in each measurement. Table I shows the crosstalk level relative to the original signal power, and the spectra of output signals with channel \#3 switched OFF are shown in Fig. 5. As shown from the figure, the crosstalk levels for OOK signals are, in general, higher than their DPSK counterparts. Moreover, FWM-induced crosstalk experienced by NRZ signals is more severe than their RZ counterparts. This result can be explained by the pulse walkoff effect between channels. As the signals are placed at wavelengths away from the zero-dispersion wavelength, the differences in group velocity between signals are
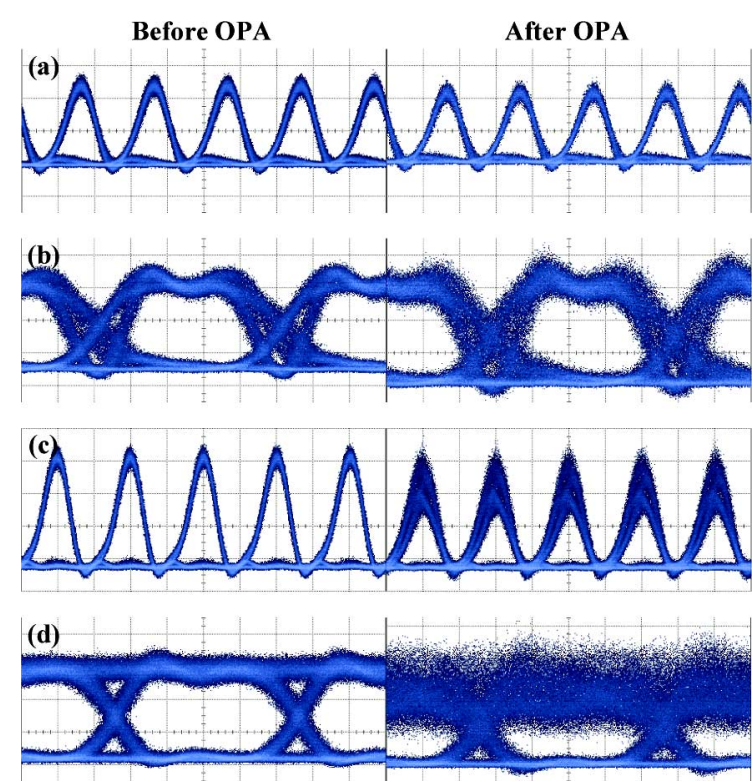

Fig. 4. Eye diagrams of channel \#1 signals with four channels, $200 \mathrm{GHz}$ separated (a) RZ-DPSK, (b) NRZ-DPSK, (c) RZ-OOK, and (d) NRZ-OOK format before and after OPA. Timescales are $50 \mathrm{ps} / \mathrm{div}$ for RZ signals and 20 ps/div for NRZ signals.

TABLE I

FWM-INDUCED CROSSTALK LEVEL

\begin{tabular}{ccccc}
\hline & $\mathrm{Ch \# 1}$ \\
& $1567.7 \mathrm{~nm}$ & $\begin{array}{c}\mathrm{Ch} \# 2 \\
1569.3 \mathrm{~nm}\end{array}$ & $\begin{array}{c}\mathrm{Ch} \# 3 \\
1570.9 \mathrm{~nm}\end{array}$ & $\begin{array}{c}\mathrm{Ch} \# 4 \\
1572.5 \mathrm{~nm}\end{array}$ \\
\hline RZ-DPSK & $-28.3 \mathrm{~dB}$ & $-22.2 \mathrm{~dB}$ & $\mathrm{NA}^{\mathrm{a}}$ & $\mathrm{NA}^{\mathrm{a}}$ \\
\hline NRZ-DPSK & $-22.5 \mathrm{~dB}$ & $-20.5 \mathrm{~dB}$ & $-28.1 \mathrm{~dB}$ & $-28.1 \mathrm{~dB}$ \\
\hline RZ-OOK & $-26.0 \mathrm{~dB}$ & $-22.0 \mathrm{~dB}$ & $-32.0 \mathrm{~dB}$ & $-27.2 \mathrm{~dB}$ \\
\hline NRZ-OOK & $-22.5 \mathrm{~dB}$ & $-18.4 \mathrm{~dB}$ & $-28.3 \mathrm{~dB}$ & $-26.2 \mathrm{~dB}$ \\
\hline
\end{tabular}

not negligible, which give rise to pulse walkoff between signal waves, especially after the decorrelation fiber in the setup or transmission fiber in field deployment. When the pulse widths of signals decrease from one-bit period (NRZ) to a fraction of bit period (RZ), the portion that signal pulses in different channels overlap decreases given the same amount of walkoff in the decorrelation fiber. As a result, the FWM efficiencies between channels also decreases [24]. This means choosing the RZ-DPSK format can also suppress FWM-induced crosstalk effectively. In addition, as seen in Fig. 5, there are two distinct FWM tones at $1570.9 \mathrm{~nm}$ and $1571.1 \mathrm{~nm}$ in the OOK spectra, while only one FWM tone at $1571.1 \mathrm{~nm}$ is observable in the NRZ-DPSK spectrum. The tone at $1571.1 \mathrm{~nm}$, which is inherited from the pump-signal FWM, does not collide with channels. However, the tone at $1570.9 \mathrm{~nm}$, which was generated by signal-signal FWM, lay on the signal grid. The disappearance of signal-signal FWM tone in NRZ-DPSK signal spectrum was due to the fact that DPSK signals had lower peak power than their OOK counterparts, which resulted in reduction of signalsignal FWM efficiency between channels. This implies DPSK signals suffered only from high-frequency $(>20 \mathrm{GHz})$ beat noise that could be suppressed by postdetection filtering, while the inband beat noise of OOK signals could not be removed by electrical filters. 

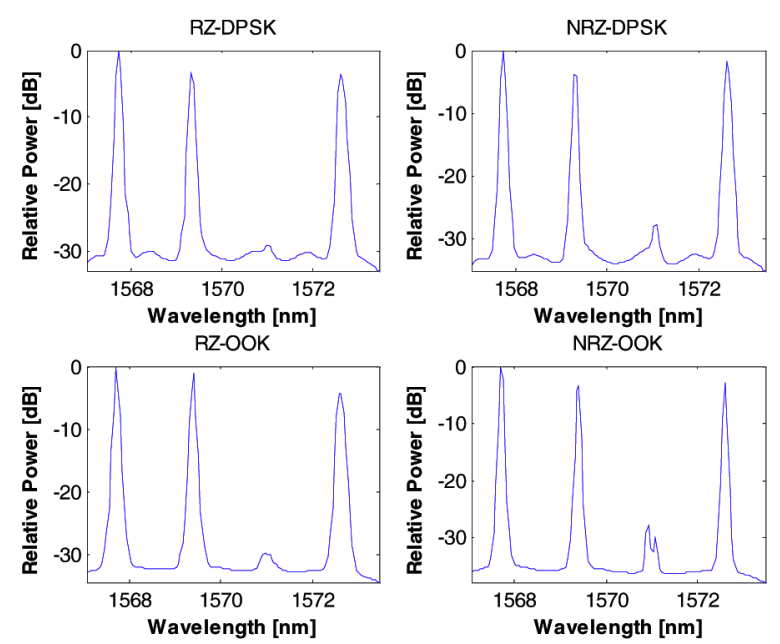

Fig. 5. Spectra of post-OPA signals with channel \#3 switched OFF.

To quantify the advantage of using RZ-DPSK over other modulation formats, bit-error rates (BERs) of signals at channel \#1 before and after OPA were compared. In order to exclude the power loss in the MZDI for DPSK demodulation, the received powers of both signals were measured at the input to the photodetector instead of MZDI for DPSK signals. Fig. 6 shows the BER plots for signals modulated with different formats. From Fig. 6(a), the power penalty at BER of $10^{-9}$ for the RZ-DPSK signal is less than that for the RZ-OOK signal with a margin of about $0.8 \mathrm{~dB}(0.8 \mathrm{~dB}$ vs. $1.6 \mathrm{~dB})$. The sensitivity gain margin is expected to increase if more channels are present at the input, which will be verified in Section IV-B. It is because the XGMinduced crosstalk, which is experienced by the OOK signal but not by the DPSK signal, will become more severe with higher total signal power. On the other hand, the NRZ-DPSK signal, which has a power penalty of $2.3 \mathrm{~dB}$, clearly outperformed NRZOOK, which failed to sustain error-free reception after OPA, as shown in Fig. 6(b). Moreover, when RZ and NRZ formats are compared, the advantage of using RZ formats is eminent. This could be attributed by the differences in FWM-induced crosstalk level when RZ and NRZ signals are compared, as in Table I. Furthermore, the pre-OPA BER plots suggest that the RZ-DPSK signals have similar sensitivity as the RZ-OOK signals, and the NRZ-DPSK signal has worse sensitivity than NRZ-OOK signal with this experimental setup. This was because only a singleended detector was used in the receiver, and the pre-OPA BER of the signals was measured at the output of the $9 \mathrm{~km} \mathrm{SMF}$ in order to exclude the chromatic dispersion-induced penalty in the decorrelation fiber from the power penalty in the OPA. As DPSK signals are more vulnerable to chromatic dispersioninduced distortion than OOK signals [26], the performance advantage of DPSK signals over OOK signals is overridden after SMF.

In addition to the comparison of modulation formats at the same average power level, a comparison of their robustness in the OPA at the same peak power level was also performed through numerical simulation using the commercial application OptSim. The wavelengths of signals and pump chosen in sim-
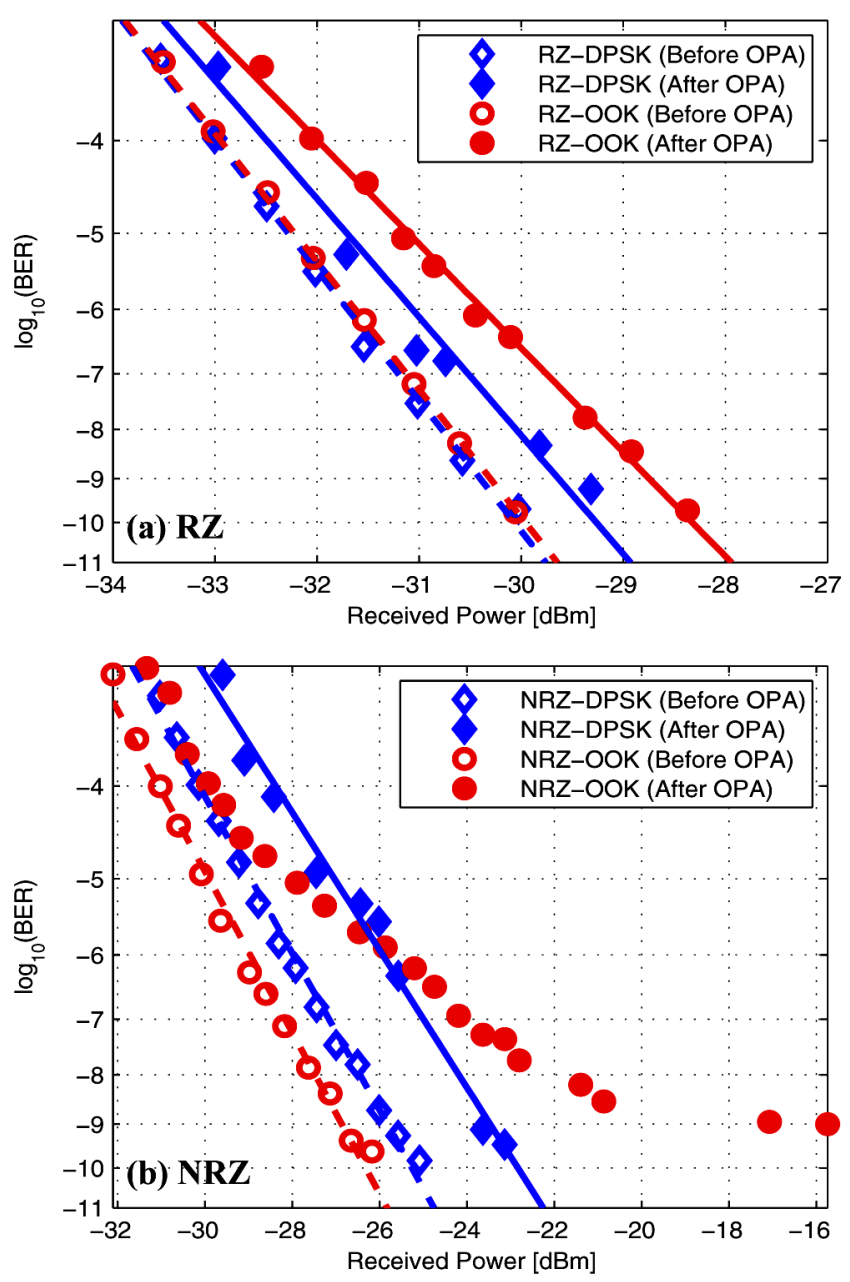

Fig. 6. Measured BER of the signal at channel 1 versus received power for (a) RZ signals and (b) NRZ signals. Empty markers with dash lines represent signals before OPA, and filled markers with solid regression lines represent signals after OPA.

ulation were the same as those used in the experiment, while the fiber parameters were adjusted to match the OPA gain spectrum obtained in the experiment. Fig. 7 shows the eye diagrams of post-OPA signals with different modulation formats at peak input power levels of $-15,-3$, and $5 \mathrm{dBm}$. The distortion observed in the pre-OPA NRZ-DPSK signal is due to chromatic dispersion in the decorrelating fiber. A similar phenomenon has also been observed in the eye diagram obtained in an experiment in Fig. 4. In a low-power regime $(-15 \mathrm{dBm})$, the signal quality is limited by the OSNR, and no crosstalk distortion is observable. As the power level rises to $3 \mathrm{dBm}$, distortion is visible from the eye diagrams of OOK signals, while no significant distortion is seen in DPSK signals. As OOK signals can suffer from both XGM- and FWM-induced crosstalk while DPSK signals suffer only from the latter, the difference between the OOK and DPSK signal quality suggests that the XGM effect has lower threshold than FWM effect. At high-power level (5dBm), the eyes of both NRZ signals are closed due to both XGM and FWM effects for the NRZ-OOK signal and purely FWM effects for the NRZ-DPSK signal. However, formats with RZ pulse shape are able to maintain wider eye opening than their NRZ 


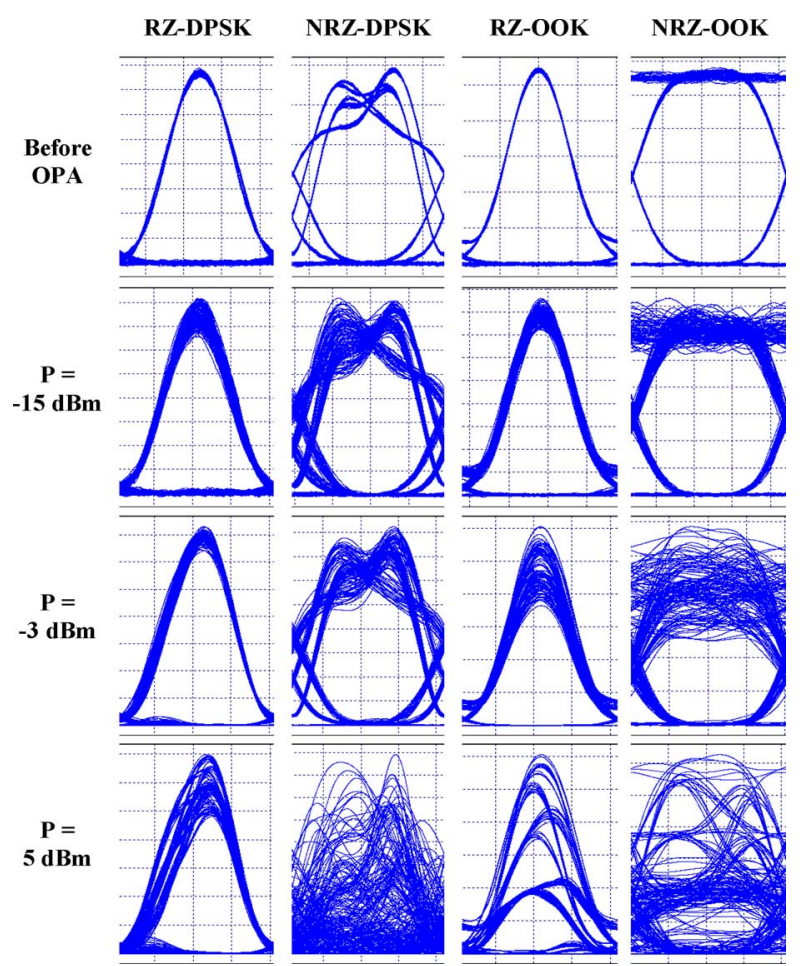

Fig. 7. Simulated eye diagrams of channel \#1 signals before and after OPA. Power levels labeled on the leftmost column indicate the peak power of the input signals. Timescales are $25 \mathrm{ps} / \mathrm{div}$.

counterparts. This is because the subunity duty ratio of these formats shortens the interaction length between signal pulses at different channels. This means RZ formats should be more robust in OPA, especially when the signal-signal FWM is likely to occur such as with high-channel-count DWDM input. Combining the advantages of pattern-independent power and subunity duty ratio, the RZ-DPSK should outperform other modulation formats in robustness to crosstalk. In the next section, we further the comparison on two RZ formats to the case with high channel-count DWDM configuration in order to prove the robustness of the DPSK format in OPA with standard DWDM configuration.

\section{B. Further Studies With Narrow Channel Spacing}

Although we have shown that the RZ-DPSK modulation format is more robust to crosstalk in the OPA when channel spacing is wide $(200 \mathrm{GHz})$, its advantage over other formats, especially RZ-OOK, would be uncertain with standard DWDM channel spacing $(100 \mathrm{GHz})$ and high channel count as they have comparable tolerance to FWM-induced crosstalk.

In order to testify the advantage of using the RZ-DPSK format, the setup of the transmitter module in Fig. 3 was altered. Fig. 8 shows the setup of the transmitter module for RZ-DPSK and RZ-OOK comparison with eight DWDM channels. Eight signal laser sources (SLD1-8) with wavelengths from 1542.9 to $1548.5 \mathrm{~nm}$ were combined by an arrayed waveguide grating (AWG1) with channel spacing of $100 \mathrm{GHz}$, and intensity modulated with $10-\mathrm{GHz}$ clock signal at the amplitude modu-
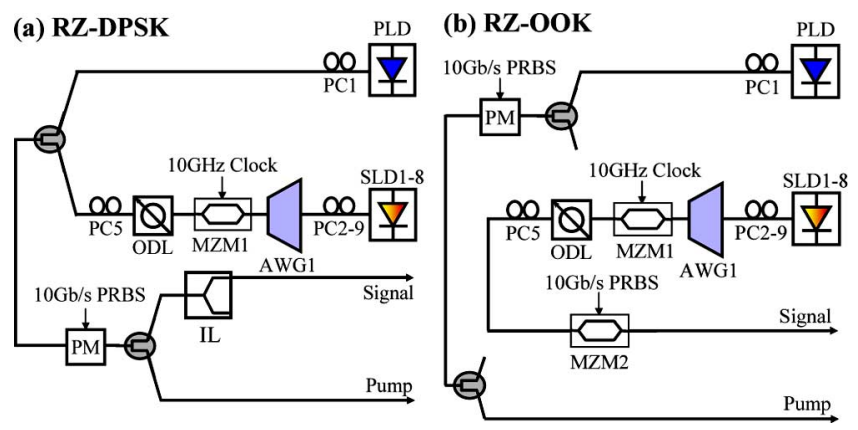

Fig. 8. Eight DWDM channel transmitter modules with (a) RZ-DPSK and (b) RZ-OOK modulation format.

lator (MZM1) for RZ pulse carving. The signal pulse trains were then modulated in different transmitters. In the RZ-DPSK transmitter, as shown in Fig. 8(a), the signal waves were combined with the pump wave at $1560.2 \mathrm{~nm}$ from a DFB laser source (PLD) and phase-modulated with $10 \mathrm{~Gb} / \mathrm{s} 2^{7}-1$ PRBS at phase modulator (PM) for data modulation of signal waves and SBS suppression of pump wave. A Shorter PRBS pattern was chosen here to further enhance the SBS threshold of the pump. The pulse trains from MZM1 were aligned to the modulating signal fed into PM by a tunable ODL. To remove the pump wave from the signal path, a 50-GHz interleaver (IL) was connected to the signal port of the 50/50 coupler after the PM. In the RZ-OOK transmitter, as shown in Fig. 8(b), the signal pulse trains after MZM1 were intensity modulated with $10 \mathrm{~Gb} / \mathrm{s}$ $2^{7}-1$ PRBS by another amplitude modulator MZM2 for data modulation, while the pump wave was phase-modulated with $10 \mathrm{~Gb} / \mathrm{s} 2^{7}-1$ PRBS at PM for SBS suppression. The unused couplers in RZ-OOK transmitter were not removed to maintain the OSNR of the pump, and therefore, the noise figure of the OPA. The modulated signals were then amplified to total power level of $4 \mathrm{dBm}$, as shown in Fig. 2. In order to extend the OPA gain bandwidth to cover all channels, the pump power at the HNL-DSF input was increased to $28 \mathrm{dBm}$. The signal gain attained was $15 \mathrm{~dB}$, which was limited by gain saturation due to high ASE noise power from pump EDFA.

The eye diagrams of RZ-DPSK and RZ-OOK signals before and after OPA are shown in Fig. 9. As observed from the eye diagrams, the RZ-OOK signal is distorted by strong XGMinduced crosstalk, as indicated by multiple-mark-level features in both Fig. 9(c) and (d), and also in less extent by FWM-induced crosstalk, as shown by the noisy mark level. On the contrary, clear eye opening is still observed for the post-OPA RZ-DPSK signal for both $\mathrm{Ch \# 4}$ and $\mathrm{Ch \# 5} \mathrm{(Fig.} \mathrm{9(a)} \mathrm{and} \mathrm{(b),} \mathrm{respectively).}$ Fig. 10. shows the $Q$-factor penalty for RZ-DPSK and RZ-OOK signals. The $Q$ factor is defined as

$$
Q(\mathrm{~dB})=10 \log _{10}(Q(\text { linear }))=10 \log _{10}\left(\frac{\bar{V}_{\mathrm{HIGH}}-\bar{V}_{\mathrm{LOW}}}{\sigma_{\mathrm{HIGH}}+\sigma_{\mathrm{LOW}}}\right)
$$

where average signal levels $V_{\mathrm{HIGH} / \mathrm{LO}}$ and noise levels $\sigma_{\mathrm{HIGH} / \mathrm{LOW}}$ are obtained from eye measurements in digital communication analyzer (DCA), and the $Q$-factor penalty is the difference in $Q$ (in decibells) between the signal before and 


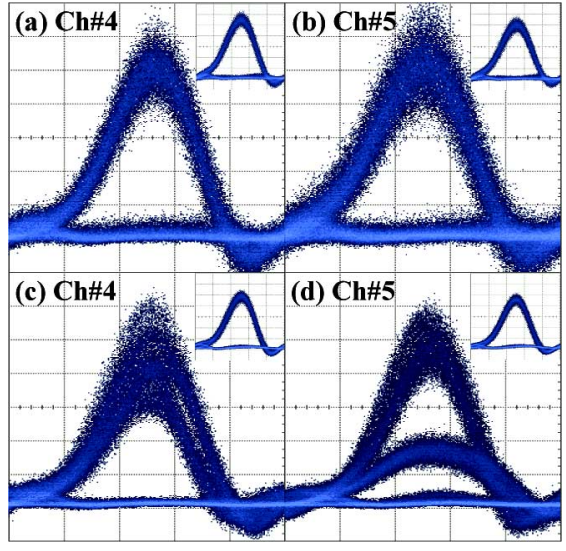

Fig. 9. Eye diagrams for RZ-DPSK (top row) and RZ-OOK (bottom row) signals after OPA. Insets shows the pre-OPA eye diagrams of the respective channels and modulation formats. Timescales are $20 \mathrm{ps} / \mathrm{div}$.

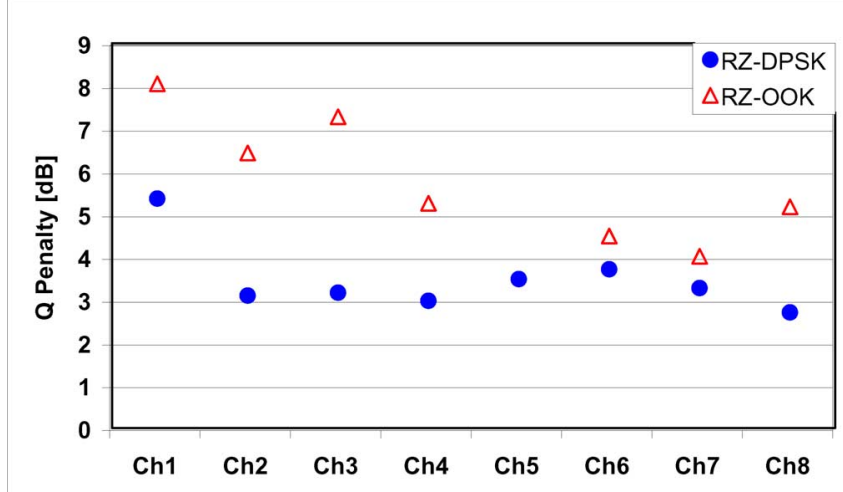

Fig. 10. $Q$-factor penalty of RZ-DPSK and RZ-OOK signals at different channels.

after OPA. On average, the $Q$-factor penalty for the RZ-DPSK signal is $2.4 \mathrm{~dB}$ less than that for the RZ-OOK signal. The higher $Q$-factor penalty of channel \#1 signals observed from the plot is mainly due to lower signal power launched at channel \#1 to equalize the channel power after signal EDFA, and higher ASE noise from pump EDFA at shorter wavelength regime. Also, the data point of channel \#5 RZ-OOK signal is missing from the plot as the $Q$-factor of the post-OPA signal is not measurable. As shown in Fig. 9(d), the strong XGM effect experienced in channel \#5 rendered the mark level undefined for this channel.

To quantify the robustness of the RZ-DPSK modulation format with multiple channels coexisting at the input to OPA, we have also measured the $Q$-factor penalty at channel \#1, \#4, and \#8 versus the number of input channels, and the results are shown in Fig. 11. The measurement was done by switching OFF signal channels sequentially starting from channel \#1, or \#2 when channel \#1 was the channel of interest. As from the plot, the variation of the $Q$-factor with different number of channels for RZ-DPSK signals is significantly less than RZ-OOK signals. From this result, it can be deduced that the crosstalk among RZ-DPSK modulated channels is less severe than its RZ-OOK counterpart. Moreover, as the penalty for the RZ-DPSK signal

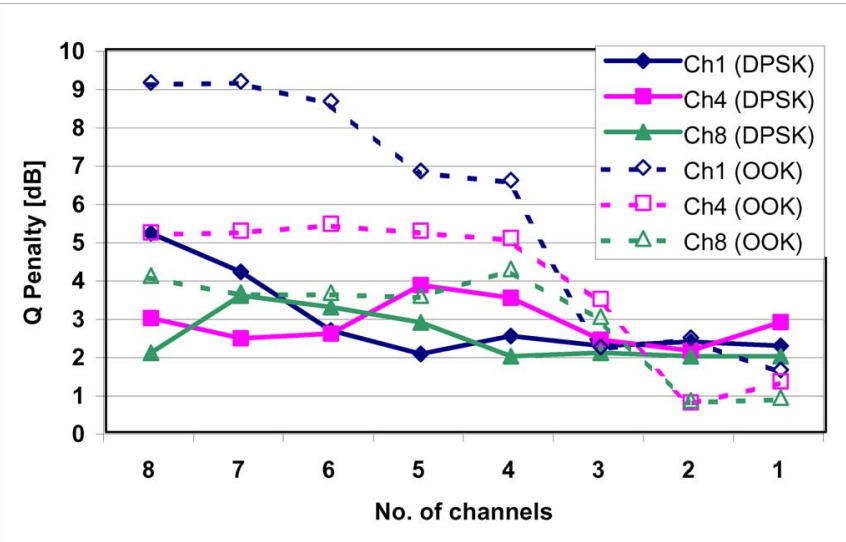

Fig. 11. $Q$-factor penalty of RZ-DPSK and RZ-OOK signals versus the number of channels.

is similar for single-channel and multiple-channel inputs, we believe that the FWM-induced crosstalk is insignificant, and the major source for the crosstalk experienced by the RZ-OOK signal is XGM.

Finally, note that the signal input power of the WDM channels used in various experimental setups was relatively high $(\sim-4.2$ $\mathrm{dBm} /$ channel in Section IV-A and $\sim-5 \mathrm{dBm} /$ channel in Section IV-B with a signal gain of $18 \mathrm{~dB}$ and $15 \mathrm{~dB}$, respectively) when compared to a practical system with about $-20 \mathrm{dBm}$ [27]. As the RZ-DPSK format is effective for suppressing nonlinear crosstalk in OPA even under such stringent conditions, we believe it will work equally well in practical environments, and furthermore, it is possible to accommodate more channels.

\section{CONCLUSION}

We experimentally demonstrated crosstalk suppression in a one-pump OPA by using the RZ-DPSK modulation format. With a 200-GHz channel spacing and a data rate of $10 \mathrm{~Gb} / \mathrm{s}$, the RZDPSK format enjoyed an improvement of at least $1 \mathrm{~dB}$ over other signals. Moreover, experimental results suggested a performance gain for RZ formats over NRZ formats in terms of the FWM-induced crosstalk level thanks to the lower duty cycle of RZ signal pulses. Even with a $100-\mathrm{GHz}$ channel spacing and eight coexisting channels, the RZ-DPSK signal still outperformed its OOK counterpart by $2.4 \mathrm{~dB}$ reduction of the $Q$-factor penalty. In addition, the difference in the $Q$-factor-penalty sensitivity between DPSK and OOK formats to the number of coexisting channels suggested that the XGM was the dominant source of crosstalk even with high channel count and dense channel spacing. These results should help to design high-performance OPAs for use in contemporary 10-Gb/s WDM communication systems.

\section{ACKNOWLEDGMENT}

The authors would like to acknowledge Sumitomo Electric Industries for providing the HNL-DSF. 


\section{REFERENCES}

[1] M. E. Marhic, K. K. Y. Wong, and L. G. Kazovsky, "Wide-band tuning of the gain spectra of one-pump fiber optical parametric amplifiers," IEEE J. Sel. Topics Quantum Electron., vol. 10, no. 5, pp. 1133-1141, Sep.-Oct. 2004.

[2] T. Torounidis, P. A. Andrekson, and B. E. Olsson, "Fiber-optical parametric amplifier with 70-dB gain," IEEE Photon. Technol. Lett., vol. 18, no. 10, pp. 1194-1196, May 2006.

[3] J. L. Blows and S. E. French, "Low-noise figure optical parametric amplifier with a continuous-wave frequency-modulated pump," Opt. Lett., vol. 27, no. 7, pp. 491-493, Apr. 2002.

[4] K. K. Y. Wong, M. E. Marhic, K. Uesaka, and L. G. Kazovsky, "Polarization-independent one-pump fiber-optical parametric amplifier," IEEE Photon. Technol. Lett., vol. 14, no. 11, pp. 1506-1508, Nov. 2002.

[5] T. Hasegawa, K. Inoue, and K. Oda, "Polarization independent frequency conversion by fiber four-wave mixing with a polarization diversity technique," IEEE Photon. Technol. Lett., vol. 5, no. 8, pp. 947-949, Aug. 1993.

[6] K. K. Y. Wong, M. E. Marhic, K. Uesaka, and L. G. Kazovsky, "Polarization-independent two-pump fiber optical parametric amplifier," IEEE Photon. Technol. Lett., vol. 14, no. 7, pp. 911-913, Jul. 2002.

[7] K. Inoue, "Polarization independent wavelength conversion using fiber four-wave mixing with two orthogonal pump lights of different frequencies," J. Lightw. Technol., vol. 12, no. 11, pp. 1916-1920, Nov. 1994.

[8] R. M. Jopson and R. E. Tench, "Polarization-independent phase conjugation of lightwave signals," Electron. Lett., vol. 29, no. 25, pp. 2216-2217, Dec. 1993.

[9] T. Torounidis, H. Sunnerud, P. Hedekvist, and P. A. Andrekson, "Amplification of WDM signals in fiber-based optical parametric amplifiers," IEEE Photon. Technol. Lett., vol. 15, no. 8, pp. 1061-1063, Aug. 2003.

[10] K. Krastev and J. Rothman, "Crosstalk in fiber parametric amplifier," presented at the Eur. Conf. Opt. Commun., Amsterdam, Netherlands, 2001, Paper We.L.3.5.

[11] P.-F. Hu and J. L. Blows, "Four-wave mixing crosstalk in optical fiber parametric amplifiers with orthogonal pumps," Opt. Commun., vol. 250, no. 4-6, pp. 421-427, Jun. 2005.

[12] J. L. Blows and P.-F. Hu, "Cross-talk-induced limitations of two-pump optical fiber parametric amplifiers," J. Opt. Soc. Amer. B, Opt. Phys., vol. 21, no. 5, pp. 989-995, May 2004.

[13] J. Blows, "Cross talk in a fibre parametric wavelength converter," presented at the Opt. Fiber Commun. Conf., Atlanta, 2003, Paper TuT4.

[14] K. K. Y. Wong, G.-W. Lui, and L.-K. Chen, "Experimental study of the WDM signal crosstalk in two-pump fiber optical parametric amplifiers," Opt. Commun., vol. 270, no. 2, pp. 429-432, Feb. 15, 2007.

[15] K. K. Y. Wong, G. W. Lu, and L. K. Chen, "Polarization-interleaved WDM signals in a fiber optical parametric amplifier with orthogonal pumps," Opt. Express, vol. 15, no. 1, pp. 56-61, Jan. 2007.

[16] T. Ito, T. Ono, Y. Yano, K. Fukuchi, H. Yamazaki, M. Yamaguchi, and K. Emura, "Feasibility study on over $1 \mathrm{bit} / \mathrm{s} / \mathrm{Hz}$ high spectral efficiency WDM with optical duobinary coding and polarization interleave multiplexing," presented at the Opt. Fiber Commun. Conf., Dallas, TX, Feb. 1997, Paper TuJ1.

[17] J. M. C. Boggio, J. D. Marconi, S. R. Bickham, and H. L. Fragnito, "Spectrally flat and broadband double-pumped fiber optical parametric amplifiers," Opt. Express, vol. 15, no. 9, pp. 5288-5309, Apr. 2007.

[18] F. A. Callegari, J. M. Chavez Boggio, and H. L. Fragnito, "Spurious four-wave mixing in two-pump fiber-optic parametric amplifiers," IEEE Photon. Technol. Lett., vol. 16, no. 2, pp. 434-436, Feb. 2004.

[19] P. S. Cho and J. B. Khurgin, "Suppression of cross-gain modulation in SOA using RZ-DPSK modulation format," IEEE Photon. Technol. Lett., vol. 15, no. 1, pp. 162-164, Jan. 2003.

[20] P. Devgan, R. Tang, V. S. Grigoryan, and P. Kumar, "Highly efficient multichannel wavelength conversion of DPSK signals," J. Lightw. Technol., vol. 24 , no. 10 , pp. $3677-3682$, Oct. 2006.

[21] B. P. P. Kuo, P. C. Chui, and K. K. Y. Wong, "Suppression of WDM signal crosstalk in fiber optical parametric amplifier by using RZ-DPSK modulation format," presented at the Opt. Fiber Commun. Conf., Anaheim, CA, Mar. 2007, Paper OWB3.

[22] B. P. P. Kuo, P. C. Chui,, and K. K. Y. Wong, "Comparison on crosstalk tolerance of RZ-DPSK and RZ-OOK modulation format in fiber optical parametric amplifier," presented at the Eur. Conf. Opt. Commun., Berlin, Germany, Sep. 2007, Paper Tu4.5.6.

[23] N. Alic, R. M. Jopson, J. Ren, E. Myslivets, R. Jiang, A. H. Gnauck, and S. Radic, "Impairments in deeply-saturated optical parametric ampli- fiers for amplitude- and phase-modulated signals," Opt. Express, vol. 15, no. 14, pp. 8997-9008, Jul. 2007.

[24] S. Kumar, "Analysis of degenerate four-wave-mixing noise in return-tozero optical transmission systems including walk-off," J. Lightw. Technol., vol. 23, no. 1, pp. 310-320, Jan. 2005.

[25] F. Forghieri, R. W. Tkach, and A. R. Chraplyvy, "Fiber nonlinearities and their impact on transmission systems," in Optical Fiber Telecommunications IIIA, I. P. Kaminow and T. L. Koch, Eds. San Diego, CA Academic, 1997.

[26] K.-P. Ho, Phase-Modulated Optical Communication Systems. New York: Springer, 2005.

[27] J. M. C. Boggio, E. A. M. Fagotto, M. E. Marhic, F. A. Callegari, and H. L. Fragnito, "Amplification of $12 \times 10 \mathrm{~Gb} / \mathrm{s}$ WDM signals with negligible FWM crosstalk in a double-pumped fiber optical parametric amplifier," Opt. Commun., vol. 280, no. 2, pp. 468-471, Dec. 2007.

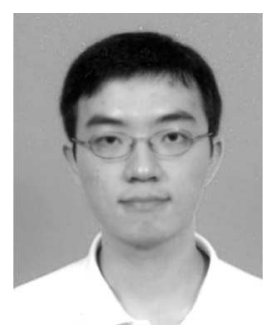

Bill Ping-Piu Kuo (S'00) received the B.Eng. degree (1st class honors) in electronic and communications engineering from the University of Hong Kong, Hong Kong, in 2006, where he is currently working toward the M. Phil degree in electronic engineering.

His current research interests include applications of fiber optical parametric and Kerr effects in optical and microwave communication systems.

P. C. Chui, photograph and biography not available at the time of publication

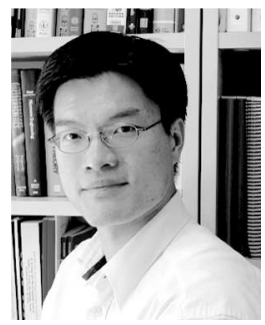

Kenneth Kin-Yip Wong (S'00-M'03) received the combined B.E. (1st class honors with medal award) degree in electrical engineering and the B.S. degree in physics from the University of Queensland, Brisbane, Q1d., Australia, in 1997, and the M.S. and $\mathrm{Ph} . \mathrm{D}$. degrees from Stanford University, Stanford, CA, in 1998 and 2003, respectively, both in electrical engineering.

He was a member of the Photonics and Networking Research Laboratory at Stanford University. During 1998-1999, he was with Hewlett-Packard Laboratories as a Research Engineer and contributed in projects that included parallel optics and vertical-cavity surface-emitting laser (VCSEL). He also worked as an Independent Consultant with Innovation CORE (a Sumitomo Electric Company), CA, in 2004. He is currently an Assistant Professor in the Department of Electrical and Electronic Engineering, University of Hong Kong, Hong Kong. He is the author or coauthor of more than 50 journal and conference papers. His past research interests include dense wavelength-division multiplexing (DWDM) systems, subcarrier multiplexed (SCM) optical systems, fiber nonlinearity, fiber optical parametric amplifiers, and photonic crystal fibers.

Dr. Wong is a member of The International Society for Optical Engineers (SPIE) and the IEEE Lasers and Electro-Optic Society (LEOS). He was the recipient of the Optical Society of America (OSA) New Focus Student Award and the IEEE/Lasers and Electro-Optics (LEOS) Graduate Student Fellowship, both in 2003. He was also the recipient of the Best Teacher Award in 20052006 from the University of Hong Kong. He is the Reviewer for Optics Letters, Journal of the Optical Society of America B, Optical Physics, Optics Express, IEEE PHOTONICS TECHNOLOGY LETTERS, IEEE/OSA JOURNAL OF LIGHTWAVE TECHNOLOGY, IEE Electronics Letters, and Optics Communications. 